\title{
Butyric acid attenuates intestinal inflammation in murine DSS-induced colitis model via milk fat globule-EGF factor 8
}

\author{
Tsuyoshi Mishiro ${ }^{1}$, Ryusaku Kusunoki ${ }^{1}$, Aya Otani ${ }^{1}$, Md Mesbah Uddin Ansary $^{1}$, Miki Tongu ${ }^{2}$, Nanae Harashima ${ }^{3}$, \\ Takaya Yamada ${ }^{2}$, Shuichi Sato ${ }^{4}$, Yuji Amano ${ }^{4}$, Kazuhito Itoh ${ }^{5}$, Shunji Ishihara ${ }^{1}$ and Yoshikazu Kinoshita ${ }^{1}$
}

Butyric acid, a short-chain fatty acid and one of the main metabolites of intestinal microbial fermentation of dietary fiber, has been shown to have an important role in maintaining the integrity of the intestinal mucosa, while it also has been shown to exert potent anti-inflammatory effects both in vitro and in vivo. However, the precise mechanisms underlying those effects have not been fully identified. We exposed colonic epithelial cells to butyric acid, then extracted total RNA samples, and subsequently hybridized them to microarray chips. Among the upregulated genes, milk fat globuleepidermal growth factor 8 (MFG-E8) was elevated by approximately fivefold. We previously reported that the potential therapeutic benefits of MFG-E8 in intestinal tissue injury were dependent not only on enhanced clearance of apoptotic cells but also required diverse cellular events for maintaining epithelial integrity. The influence of butyric acid on cell function is often attributed to its inhibition of histone deacetylases (HDACs). We found that acetylation on histone 3 lysine 9 (acetyl-H3K9) around the MFG-E8 promoter was significantly increased with butyric acid exposure. Experimental colitis was induced by administration of dextran sodium sulfate (DSS) in C57BL/6N (MFG-E8 ${ }^{+1+}$ ) and MFG-E8 ${ }^{-1-}$ mice. Although the colonic bacterial compositions in wild-type (WT) and MFG-E8 ${ }^{-1}$ mice were not significantly different, intrarectal administration of butyric acid during an acute phase of colitis attenuated intestinal inflammatory parameters and inhibited body weight loss in the WT mice. Our novel findings suggest that butyric acid has significant antiinflammatory effects partly via MFG-E8 on DSS-induced murine experimental colitis.

Laboratory Investigation (2013) 93, 834-843; doi:10.1038/labinvest.2013.70; published online 10 June 2013

KEYWORDS: apoptosis; butyric acid; intestinal inflammation; milk fat globule-epidermal growth factor 8; 16S rRNA sequence

The cells of hundreds of different kinds of microbes far exceeding the total number of cells that comprise human tissues exist in the gastrointestinal tract, where they shape enteric microflora (gut microbiota) and build an original metabolism system. It is also becoming clear that these microbes have great influence on physiological functions as well as onset of disease, especially obesity and inflammatory bowel disease. ${ }^{1-4}$ Short-chain fatty acids (SCFAs), primarily acetate, propionate, and butyrate, are organic acids produced within the intestinal lumen by microbial fermentation of mainly undigested dietary carbohydrates and the major energy source for colonic epithelial cells. SCFAs have important roles in mucosal cell proliferation, cell differentiation, stimulation of the luminal autonomic nervous systems, and a variety of intestinal mucosal immune regulations. ${ }^{5}$ In particular, butyrate has been reported to have inhibitory effects on histone deacetylases (HDACs) and is also thought to have anti-inflammatory effects. ${ }^{6}$ Disease activity related to rat experimental colitis induced by dextran sodium sulfate (DSS) has been demonstrated to be significantly suppressed by butyrate enema treatments. ${ }^{7,8}$ In humans, butyrate enemas are used as therapy for inflammatory bowel disease as well as a variety of other diseases characterized by colonic inflammation. ${ }^{9-11}$ However, the detailed mechanisms

\footnotetext{
${ }^{1}$ Department of Gastroenterology and Hepatology, Faculty of Medicine, Shimane University, Izumo, Shimane, Japan; ${ }^{2}$ Department of Experimental Animals, Center for Integrated Research in Science, Faculty of Medicine, Shimane University, Izumo, Shimane, Japan; ${ }^{3}$ Department of Immunology, Faculty of Medicine, Shimane University, Izumo, Shimane, Japan; ${ }^{4}$ Division of Gastrointestinal Endoscopy, Shimane University Hospital, Izumo, Shimane, Japan and ${ }^{5}$ Faculty of Life and Environmental Science, Shimane University, Matsue, Shimane, Japan

Correspondence: Dr T Mishiro, MD, PhD, Department of Gastroenterology and Hepatology, Faculty of Medicine, Shimane University, 89-1, Enya-cho, Izumo, Shimane 6938501, Japan.
}

E-mail: tmishiro@med.shimane-u.ac.jp

Received 19 October 2012; revised 15 March 2013; accepted 15 April 2013 
initiated by butyrate related to its protective effects have not been completely elucidated.

Milk fat globule-epidermal growth factor 8 (MFG-E8), also termed lactadherin, BA46, or SED1, is a glycoprotein secreted from different cell types that participates in phagocytosis of apoptotic cells by forming a link between phosphatidylserine on apoptotic cells and $\alpha_{\mathrm{v}} \beta_{3}$-integrin on phagocytes. ${ }^{12,13}$ Several studies have revealed that MFG-E8 has important roles in the pathogenesis of inflammatory and autoimmune diseases, as well as sepsis, atherosclerosis, and neurodegenerative disorders, ${ }^{14-16}$ whereas recent findings have also revealed that MFG-E8 mRNA and protein levels were lower in inflamed intestinal specimens obtained during biopsies from patients with ulcerative colitis (UC) as compared with control biopsy specimens. ${ }^{17}$ Those features have been suggested to be dependent not only on enhanced clearance of apoptotic cells but also on various novel molecular mechanisms. ${ }^{18,19}$ We recently showed a beneficial anti-inflammatory role of intrarectal administration of recombinant MFG-E8 protein in a murine model of colitis. ${ }^{20}$ Although MFG-E8 is expressed in a wide variety of cell types to participate in several cellular events and modulates immune responses in numerous conditions, its role and the mechanism underlying those functions have not been clearly defined.

In this study, we employed intrarectal administration of butyrate in a model of acute colitis to elucidate its effects by evaluating colitis parameters. Furthermore, we explored the therapeutic role of butyrate via MFG-E8 in a murine experimental colitis model.

\section{MATERIALS AND METHODS Cell Culture Conditions}

NCI-H716, HCT-15, and THP-1 cells were obtained from American Type Culture Collection (ATCC, Manassas, VA, USA) and cultured in RPMI-1640 medium (Sigma-Aldrich), supplemented with $10 \%(\mathrm{v} / \mathrm{v})$ fetal bovine serum (ICN Biomedicals, Aurora, OH, USA) and penicillin/streptomycin (Gibco). Cells were kept at $37^{\circ} \mathrm{C}$ in an incubator under a humidified condition containing $5 \% \mathrm{CO}_{2}$.

\section{Reagents and Antibodies}

DSS (5 kDa; Wako Pure Chemicals), sodium butyrate (Sigma-Aldrich), trichostatin A (Sigma-Aldrich), an RNeasy Mini Kit (Qiagen), a QIAamp DNA Stool Mini Kit (Qiagen), a MinElute PCR Purification Kit (Qiagen), and enzyme-linked immunosorbent assay (ELISA) kits for mouse interleukin (IL)-1 $\beta$, IL-6, CXCL2/macrophage inflammatory protein (Mip)-2 (R\&D Systems), MFG-E8, $\mathrm{TNF} \alpha$ (Abcam), myeloperoxidase (MPO) (Cusabio Biotech), and human MFG-E8 (Cusabio Biotech) were acquired from their respective suppliers. The antibodies used were anti-histone H3 (acetyl K9) (Abcam) and control IgG (Santa Cruz).

\section{SCFA Concentration in Cecal Contents}

Cecal contents were removed immediately after performing a laparotomy, and then SCFAs (succinic acid, acetic acid, propionic acid, butyric acid) were measured using HPLC. Briefly, after the addition of an internal control, distilled water was added to produce $200 \mathrm{mg}$ of each sample. Following incubation at $80^{\circ} \mathrm{C}$ for $15 \mathrm{~min}$, mechanical shaking with beads and centrifugation at 13000 r.p.m. were performed for $10 \mathrm{~min}$, and then the supernatant was filtered through a membrane filter (pore size $0.45 \mu \mathrm{m}$ ). SCFAs in the samples were then separated using a Shim-pack SCR-102 (H) (Shimadzu Corporation, Kyoto, Japan). For HPLC, the column temperature was $45^{\circ} \mathrm{C}$ and the mobile phase was $5 \mathrm{mmol} / \mathrm{l}$ p-toluenesulfonic acid (flow rate: $0.8 \mathrm{ml} / \mathrm{min}$ ). A CDD-10A (Shimadzu Corporation) was used for detection.

\section{DNA Preparations from Mouse Stool Samples}

DNA was extracted from mouse stool samples (cecal contents) using a QIAamp DNA Stool Mini Kit (Qiagen), according to the manufacturer's protocol. The amount of DNA was determined using NanoDrop (ND-1000) and its integrity was checked with $1 \%$ agarose gel electrophoresis after staining the gels with ethidium bromide. DNA was then dissolved in $200 \mu \mathrm{l}$ of Tris-EDTA buffer (pH 8) and the resultant preparations were used as template DNAs in the PCR steps.

\section{PCR-DGGE for 16S rRNA Gene}

$16 \mathrm{~S}$ rRNA gene fragments were amplified from stool DNA with the forward primer HDA1-GC clamp and the reverse primer HDA2 ${ }^{21}$ (Supplementary Table 2). PCR reactions were performed for $4 \mathrm{~min}$ at $94{ }^{\circ} \mathrm{C}$, with 30 cycles of $30 \mathrm{~s}$ at $94^{\circ} \mathrm{C}, 30 \mathrm{~s}$ at $56^{\circ} \mathrm{C}$, and $1 \mathrm{~min}$ at $68^{\circ} \mathrm{C}$, and then a final step for $7 \mathrm{~min}$ at $68^{\circ} \mathrm{C}$. The reactions contained $5 \mu \mathrm{l}$ of $10 \times$ reaction buffer, $2.5 \mathrm{mM}$ of each dNTP, 10 pmol of each primer, $0.5 \mathrm{U}$ of Pyrobest DNA Polymerase (Takara Bio, Shiga, Japan), $500 \mathrm{ng}$ of template DNA, and an adequate quantity of sterilized distilled water to produce a volume of $50 \mu \mathrm{l}$. PCR products were examined using $1 \%$ agarose gel electrophoresis, stained with ethidium bromide, and visualized by UV transillumination. PCR products were purified using a QuickStep 2 PCR Purification Kit (Edge Biosystems, Gaithersburg, MD, USA). DGGE analysis was performed using a D-Code ${ }^{\mathrm{TM}}$ universal mutation detection system (Bio-Rad, Tokyo, Japan), according to the instruction manual. A denaturing gradient gel was prepared with $10 \%$ polyacrylamide and a $30-50 \%$ denaturing gradient $(100 \%$ denaturant contained $7 \mathrm{M}$ urea and $40 \%$ formamide) in TAE buffer ( $40 \mathrm{mM}$ Tris, $20 \mathrm{mM}$ acetate, $1 \mathrm{mM}$ EDTA, pH 8.0). Electrophoresis was performed at $130 \mathrm{~V}$ for $4.5 \mathrm{~h}$ in TAE buffer at $60^{\circ} \mathrm{C}$. After electrophoresis, gels were stained with $0.1 \%$ SYBR Gold (Invitrogen) and scanned with a CCD camera system (Toyobo, Osaka, Japan). DGGE profiles were digitally processed with Multi Gauge software version 3.0 (Fuji Photo Film, Kanagawa, Japan) in a multistep procedure following the manufacturer's instructions. 
PCR Amplification and Analysis of 16S rRNA Sequences The 16S rRNA gene was PCR-amplified from stool DNA with the primer pairs described in Supplementary Table 2 using TaKaRa Ex Taq PCR mixture (Takara Bio). PCR for each method was carried out in a 2720 Thermal Cycler (Applied Biosystems, Foster City, CA, USA) with the following parameters: initial denaturation at $94^{\circ} \mathrm{C}$ for $3 \mathrm{~min}$, followed by 30 cycles of $94^{\circ} \mathrm{C}$ for $40 \mathrm{~s}, 56^{\circ} \mathrm{C}$ for $1 \mathrm{~min}$, and $72{ }^{\circ} \mathrm{C}$ for $1 \mathrm{~min}$, with a final extension step at $72{ }^{\circ} \mathrm{C}$ for $10 \mathrm{~min}$. PCR products were subjected to $1.5 \%$ agarose gel electrophoresis, and then the DNA band of the correct size was excised and purified using a WizardH SV Gel and PCR Clean-Up System (Promega, St Louis, MO, USA). Equal amounts of purified PCR products were pooled for subsequent 454 pyrosequencing. Emulsion PCR was performed using a GS Titanium SV emPCR Kit (Roche/454 Life Sciences), according to the manufacturer's instructions, whereas 454 pyrosequencing was carried out with a GS Titanium PicoTiterplate Kit (Roche/454 Life Sciences). Sequencing results were analyzed with Roche software version 2.5.3 by signal processing for amplicons.

\section{Quantitative Real-Time PCR Analysis}

Total RNA was extracted from colonic tissues and cultured cells using an RNeasy Mini Kit (Qiagen), and then equal amounts of RNA treated with DNaseI (Ambion) were reverse transcribed into cDNA using a High Capacity cDNA Reverse Transcription Kit (Applied Biosystems). A real-time fluorescence PCR assay using an ABI Stepone-plus (Applied Biosystems) based on SYBR Green (Applied Biosystems) was then performed. The threshold was set to cross a point at which the PCR amplification was linear, then the number of cycles (Ct) required to reach the threshold was determined, and finally analyzed using Microsoft Excel. The primer sequences are listed in Supplementary Table 2.

\section{Microarray Analysis: cRNA Amplification, Labeling, Hybridization, and Data Analysis}

Total RNA was isolated from NCI-H716 cells with or without butyric acid treatment $(5 \mathrm{mM})$, and used for microarray analysis. Contaminating DNA in the extracted RNA was removed using DNaseI. RNA quality was checked by denaturing gel electrophoresis, while the quantity was assessed using NanoDrop (ND-1000). Total RNA was labeled with Cy5 using an Amino Allyl MessageAMP II aRNA Amplification Kit (Applied Biosystems). Cy5-labeled aRNA was pooled and added to a hybridization buffer, and then hybridized to a 3D-Gene Human Oligo chip 25k (Toray Industries, Tokyo, Japan) for $16 \mathrm{~h}$. For efficient hybridization, this microarray is constructed in three dimensions, with a well used as the space between the probes and cylinder-stems with 70-mer oligonucleotide probes on top. Hybridization was performed according to the protocol of the supplier. Hybridization signals were scanned using a ScanArray Express Scanner (Perkin-Elmer) and processed by GenePixPro version 5.0 (Molecular Devices, Sunnyvale, CA, USA). Raw data for each spot were normalized by substitution with the mean intensity of the background signal determined by the signal intensities of all blank spots with $95 \%$ confidence intervals. Raw data intensities $>2$ standard deviations (s.d.'s) of the background signal intensity were considered to be valid. Detected signals for each gene were normalized using a global normalization method (median of detected signal intensity adjusted to 50). Genes that were upregulated are shown in Table 1.

\section{Chromatin Immunoprecipitation and Quantitative PCR Analyses}

NCI-H716 cells $\left(1 \times 10^{7}\right)$ with or without butyric acid treatment were crosslinked with $1 \%$ formaldehyde at $37^{\circ} \mathrm{C}$ for $10 \mathrm{~min}$. Crude cell lysates were sonicated to generate DNA fragments of 200-1000 bp. Chromatin immunoprecipitation (ChIP) assays were performed with anti-histone H3 (acetyl K9) antibodies as well as control IgG. ${ }^{22}$ PCR amplification was carried out for 33 cycles at $95^{\circ} \mathrm{C}$ for $30 \mathrm{~s}, 58^{\circ} \mathrm{C}$ for $30 \mathrm{~s}$, and $72{ }^{\circ} \mathrm{C}$ for $30 \mathrm{~s}$. DNA enrichment in the ChIP samples was determined by agarose gel electrophoresis and real-time PCR analysis using an ABI Stepone-plus (Applied Biosystems) and SYBR green fluorescence. The threshold was set to cross a point at which the PCR amplification was linear, and the number of cycles $(\mathrm{Ct})$ required to reach the threshold was determined and analyzed using Microsoft Excel. PCR amplifications were performed using precipitated DNA samples and input DNA. The primer sequences are listed in Supplementary Table 2.

\section{Colitis Induction and Sodium Butyrate Enemas in Mice}

Seven-week-old male-specific pathogen-free wild-type (WT) C57BL/6N (MFG-E8 ${ }^{+l+}$ ) mice (Charles River Laboratories) and MFG-E8 ${ }^{-1-}$ mice, kind gifts from Dr Shigekazu Nagata (Kyoto University, Japan), were housed according to our institutional guidelines following the approval of the Ethics Committee of Shimane University. We have been breeding WT and MFG-E8 ${ }^{-1-}$ mice in our vivarium for more than 10 generations. Generally, a single group of normal mice $(n=3)$ was euthanized to screen for tissue-specific MFG-E8 expression using a western blotting method. All mice were specific pathogen free, and maintained under a strict 12 -h light cycle (lights on at 0700 hours, off at 1900 hours) and given a regular chow diet (Harlan, diet no. 2018). The in vivo study protocol is shown in Figure 4a. To produce a DSS colitis model, a group containing five mice was fed 2.5\% DSS in drinking water for 7 days, whereas the control group received only normal drinking water throughout the experiment. Mice were anesthetized with an intraperitoneal pentobarbital injection and then given an intrarectal administration of $1 \mathrm{ml}$ of $100-\mathrm{mM}$ butyrate each day, which continued until euthanasia. Control mice received only $1 \mathrm{ml}$ of saline using the same protocol. Parameters used for colitis evaluation, including body weight, stool consistency, and rectal bleeding, were determined by visual inspection and recorded daily. After stopping intrarectal treatment, the mice 
Table 1 Genes upregulated in colon epithelial cells by butyrate acid exposure

\begin{tabular}{|c|c|c|c|c|}
\hline Known cellular function & Gene description & Gene symbol & $\begin{array}{l}\text { GenBank } \\
\text { accession no. }\end{array}$ & $\begin{array}{c}\text { Array } \\
\text { (fold induction) }\end{array}$ \\
\hline \multirow{6}{*}{ Cell adhesion molecules } & Integrin alpha 3 & ITGA3 & NM_005501 & 1.19 \\
\hline & Integrin alpha 5 & ITGA5 & NM_002205 & 2.16 \\
\hline & Phosphatase and tensin homolog & PTEN & NM_000314 & 1.88 \\
\hline & Ras GTPase-activating protein 1 & RASA1 & NM_002890 & 1.23 \\
\hline & Transforming protein $R$ hoA & RHOA & NM_001664 & 1.31 \\
\hline & Vinculin & $V C L$ & NM_014000 & 1.53 \\
\hline \multirow[t]{9}{*}{ Growth factors } & Platelet-derived endothelial cell growth factor & ECGF1 & NM_001953 & 3.56 \\
\hline & Placental growth factor & PGF & NM_002632 & 3.67 \\
\hline & Neuregulin 2 & NRG2 & NM_004883 & 1.44 \\
\hline & Transforming growth factor beta 1 & TGFB1 & NM_000660 & 1.31 \\
\hline & Inhibin beta A chain & INHBA & NM_002192 & 1.34 \\
\hline & Oxidative stress-induced growth inhibitor 1 & OSGIN1 & NM_182981 & 2.36 \\
\hline & Growth differentiation factor 11 & GDF11 & NM_005811 & 2.57 \\
\hline & Insulin-like growth factor 2 & IGF2 & NM_001007139 & 1.51 \\
\hline & Dickkopf-related protein 1 & $D K K 1$ & NM_012242 & 2.88 \\
\hline \multirow{2}{*}{ Cytokines and chemokines } & Chemokine (C-X3-C motif) ligand 1 & CX3CL1 & NM_002996 & 1.89 \\
\hline & Toll-interacting protein & TOLLIP & NM_019009 & 1.49 \\
\hline
\end{tabular}

were euthanized, the colons were dissected, and then subjected to histological procedures and an ELISA.

\section{Histological Examination}

For histology, 3- $\mu \mathrm{m}$ formalin-fixed paraffin-embedded colon tissue sections were stained with hematoxylin and eosin, and then examined under a light microscope by two investigators in a double-blinded manner. Histological grading was evaluated as described previously. ${ }^{20}$ Three different parameters were considered, severity of inflammation (based on polymorphonuclear neutrophil infiltration; 0-3: none, slight, moderate, and severe), depth of injury (0-3: none, mucosal, mucosal and submucosal, and transmural), and crypt damage (0-4: none, basal one-third damaged, basal two-thirds damaged, only surface epithelium intact, entire crypt, and epithelium lost). The score for each parameter was multiplied by a factor reflecting the percentage of tissue involvement $(\times 1,0-25 \% ; \times 2,26-50 \% ; \times 3,51-75 \%$; and $\times 4,76-100 \%)$, and then all values were added to a sum with a maximum possible score of 40 .

\section{Statistical Analysis}

All quantitative data are expressed as the mean \pm s.e. Student's $t$-test was used for statistical determinations. Values of $P<0.05$ were considered to be statistically significant.

\section{RESULTS}

DSS Treatment Induces Changes in Gut Microbiota of C57/BL6N Mice

C57/BL6N WT mice given 2.5\% DSS for 7 days showed timedependent weight loss (Figure 1a). To examine whether DSS treatment altered the established gut microbiota composi- 
a

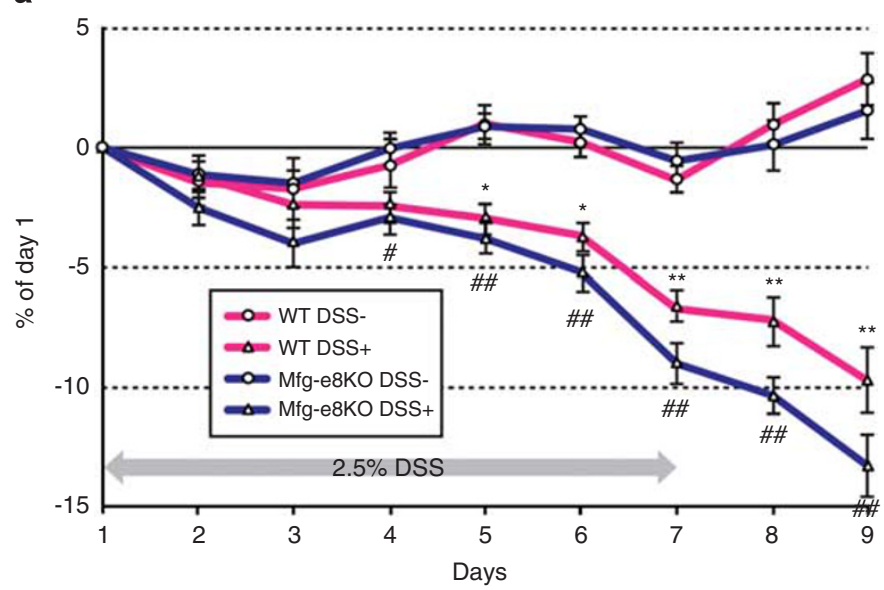

C

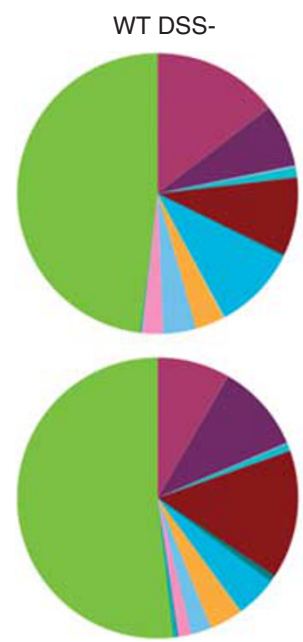

KO DSS-
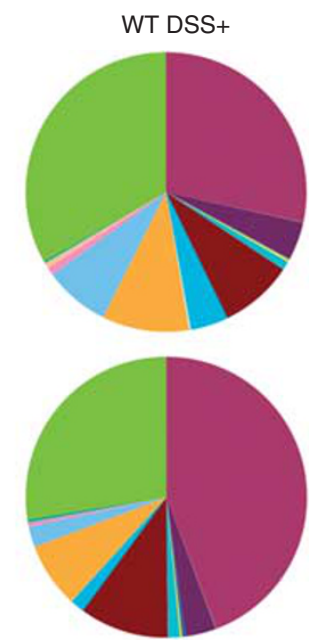

KO DSS+ b
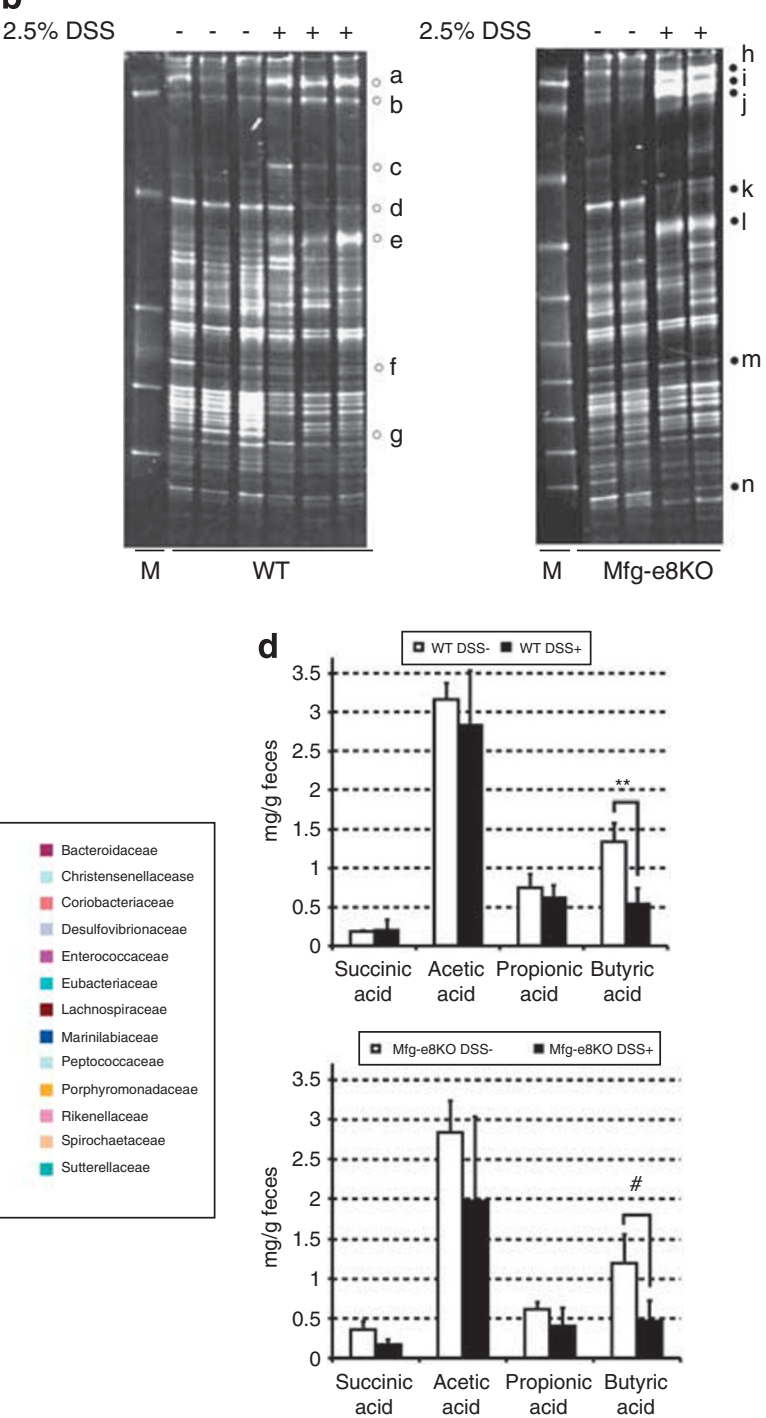

Figure 1 Dextran sodium sulfate (DSS) treatment induced changes in C57/BL6N and milk fat globule-epidermal growth factor 8 (MFG-E8) ${ }^{-1-}$ mice gut microbiota. (a) Time-course changes of body weight in 2.5\% DSS-treated C57/BL6N (wild-type (WT)) and MFG-E8 ${ }^{-1-}$ mice. Data are expressed as serial changes in the percentage of weight change during DSS administration. ${ }^{*} P<0.05,{ }^{* *} P<0.01$ vs DSS $(-)$ WT mice, ${ }^{\#} P<0.05,{ }^{\# \#} P<0.01$ vs DSS $(-)$ MFGE8 ${ }^{-I-}$ mice. Error bars indicate standard error of mean (s.e.m.) values ( $n=5$ mice per group). (b) Denaturing gradient gel electrophoresis (DGGE) gel profiles of microbiota in feces samples from control and DSS-treated WT and MFG-E8 ${ }^{-1-}$ mice. (c) Pyrosequencing analysis of cecal microbial taxa families in control and DSS-treated WT and MFG-E8 ${ }^{-1-}$ mice. Data are shown as the percentage of total identified sequences per group. (d) Shortchain fatty acid concentration in cecal contents. ${ }^{* *} P<0.01$ vs DSS (-) WT mice, ${ }^{\#} P<0.05$ vs DSS (-) MFG-E8 ${ }^{-1-}$ mice. Error bars indicate s.e.m. values ( $n=5$ mice per group). KO, knockout.

tion, we performed $16 \mathrm{~S}$ ribosomal RNA-targeted polymerase chain reaction-DGGE (Figure $1 \mathrm{~b}$, left panel and Supplementary Table 1, upper panel) and sequencing (Figure 1c, upper panel) analyses. Before DSS treatment, the DGGE profiles among the groups were similar, whereas DSS disrupted the gut microbiota composition. Sequencing analysis showed that DSS treatment increased the proportion of Bacteroidaceae (dominant genus: Bacteroides) and Porphyromonadaceae (dominant genus: Parabacteroides), whereas it decreased the Clostridiaceae (dominant genus: Clostridium) and Ruminococcaceae (dominant genus: Ruminococcus) populations. Next, to confirm changes in concentrations of stool organic acid produced by bacterial fermentation of dietary fiber, we evaluated mice with and without DSS treatment (Figure 1d, upper panel). The concentrations of succinic, acetic, and propionic acid did not change with DSS treatment, whereas that of butyric acid showed a significant decrease, suggesting the possible effects of butyric acid in the development of or recovery from DSS-induced colitis.

\section{Butyric Acid Upregulates Transcriptional Expression of MFG-E8 in Cell Lines}

To elucidate the possible role of butyric acid, we investigated genes specifically upregulated by butyric acid treatment in 
cultured cell lines. Microarray analysis was performed using mRNA from NCI-H716 cells, a colon epithelial cell line, to determine the status of gene expression after treatment with $5 \mathrm{mM}$ of butyric acid (pH 7.4 in culture medium). ${ }^{6}$ From the large number of upregulated genes identified, we chose cell adhesion molecules, potent growth factors, and cytokines that are usually involved in inflammatory bowel disease, which are presented in Table 1. In the context of inflammation, IL-8, CXCL1, and CXCL3 were significantly elevated, as reported previously. ${ }^{23}$ Moreover, MFG-E8, a cell adhesion molecule and partially potent growth factor, was upregulated nearly fivefold. To correlate the microarray results, we performed quantitative real-time PCR assays of MFG-E8 after butyric acid treatment. MFG-E8 mRNA levels were elevated by the administration of butyric acid in a dosedependent manner (Figure 2a). In addition, another colon epithelial cell line (HCT-15) and a mesenchymal cell line (THP-1) also showed significant increases in MFG-E8 mRNA levels following butyric acid administration in a time-dependent manner (Figure 2b). Similarly, we also confirmed an increased level of MFG-E8 protein in culture supernatants of the butyric acid-treated cell lines (Figure 2c).

\section{Butyric Acid Changes Histone Modifications Around MFG-E8 Promoter Regions}

The ability of butyric acid to influence cell function is considered to be related to its regulation of gene expression, which is often attributed to inhibition of HDACs. ${ }^{6}$ AcetylH3K9 is well known to be associated with active transcription. ${ }^{24}$ To investigate histone modification around the MFG-E8 promoter region, we first designated the three amplification sites as MP1, MP2, and MP3 (Figure 3a). Next, a ChIP assay followed by a quantitative real-time PCR assay was performed to evaluate acetyl-H3K9 enrichment around the promoter regions of these sites. The acetyl-H3K9 level in those sites was significantly increased in a time-dependent manner by butyric acid exposure (Figure 3b). Suberoylanilide hydroxamic acid and trichostatin $\mathrm{A}$ are also involved as HDAC inhibitors. ${ }^{25,26}$ As observed with butyric acid, trichostatin A also induced MFG-E8 expression in the colon epithelial cell lines in a time-dependent manner (Figure 3c). These results suggest that the increased expression of MFG-E8 induced by butyric acid is due to hyperacetylation around the promoter region of that growth factor.

\section{DSS Treatment Induces Changes in Gut Microbiota of MFG-E8KO Mice}

To confirm the effects of butyric acid via MFG-E8 on DSSinduced experimental colitis, we utilized MFG-E8 knockout (KO) mice. When given 2.5\% DSS in drinking water, those mice also showed time-dependent weight loss, as seen with WT mice (Figure 1a). Furthermore, 16S ribosomal RNA-targeted polymerase chain reaction-DGGE (Figure 1b, right panel and Supplementary Table 1, lower panel) and sequencing (Figure 1c, lower panel) analyses were used to examine the microbiota composition. Prior DSS treatment, the DGGE profiles were similar among the groups. DSS treatment disrupted the gut microbiota composition in a manner similar to but slightly different from the pattern seen in WT mice with and without DSS treatment. The results of pyrosequencing and $16 \mathrm{~S}$ metagenomics showed that the bacterial compositions of WT and MFG-E8KO mice without DSS treatment were not significantly different. On the other hand, DSS treatment slightly increased the proportion of Bacteroidaceae (dominant genus: Bacteroides) as compared with WT mice, whereas there were similar decreases in the Clostridiaceae (dominant genus: Clostridium) and Ruminococcaceae (dominant genus: Ruminococcus) populations. Finally, changes
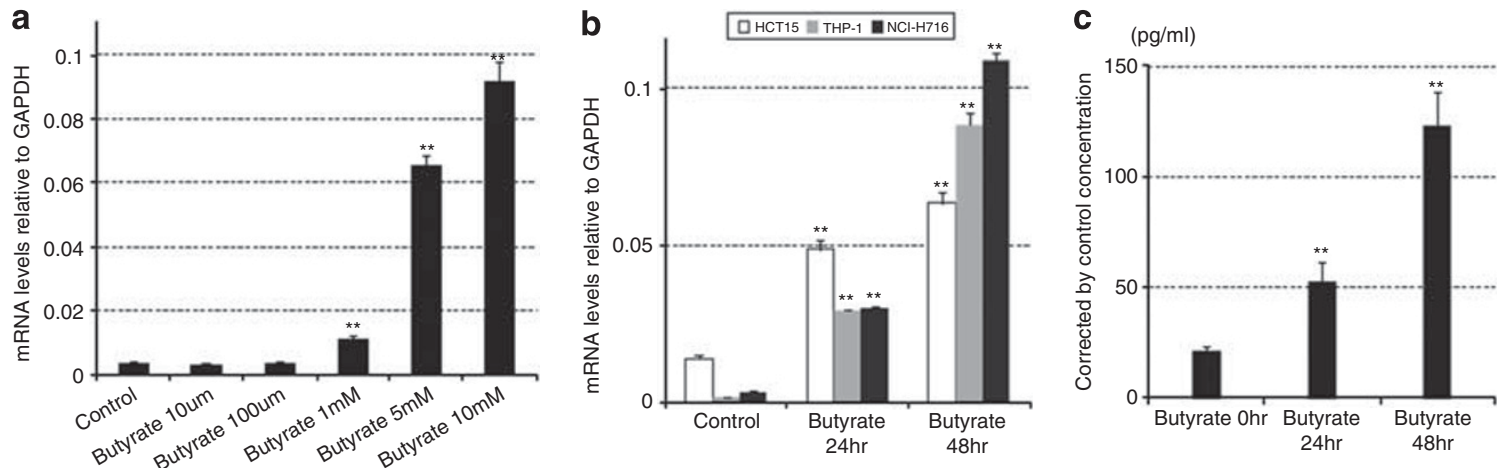

Figure 2 Butyrate acid enhanced milk fat globule-epidermal growth factor 8 (MFG-E8) expression in cell lines. (a) NCI-H716 cells were treated with different concentrations of butyrate for $48 \mathrm{~h}$. Using quantitative real-time polymerase chain reaction (PCR), the transcriptional levels of MFG-E8 were analyzed relative to that of glyceraldehyde-3-phosphate dehydrogenase (GAPDH). Values are shown as the mean and standard deviation of results from more than three independent experiments. ${ }^{*} P<0.01$ vs control (non-stimulated cells). (b) Time-dependent mRNA expression of MFG-E8 in various cell lines after stimulation with $10 \mathrm{mM}$ butyrate. The culture conditions for each cell line are described in Materials and Methods (no addition of phorbol 12-myristate 13-acetate (PMA)). All signals were standardized to the intensity of GAPDH. ${ }^{* *} P<0.01$ vs control (non-stimulated cells). (c) Time-dependent production of MFG-E8 proteins in NCl-H716 cells treated with $10 \mathrm{mM}$ butyrate acid. MFG-E8 contents in culture supernatants were measured using an enzyme-linked immunosorbent assay (ELISA). Error bars indicate the standard error of mean values obtained from more than three independent experiments. ${ }^{*} P<0.01$ vs Control (non-stimulated cells). 
a

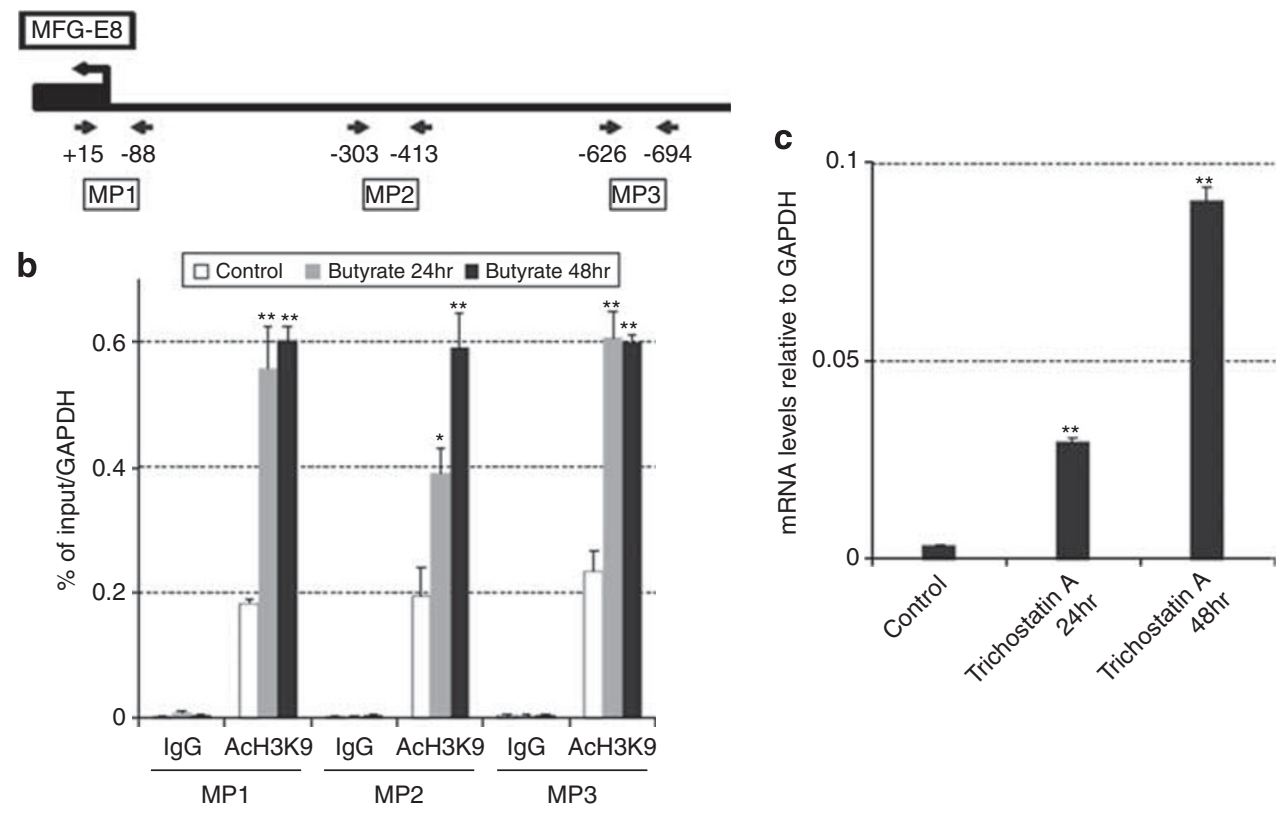

Figure 3 Accumulation of acetylated histones in chromatin of milk fat globule-epidermal growth factor 8 (MFG-E8) gene in NCI-H716 cells. (a) Schematic representation of MFG-E8 promoter from chromatin immunoprecipitation (ChIP) analysis. Three sets of PCR primers, designated as MP1, MP2, and MP3, were used for amplification of the distal and proximal MFG-E8 promoters. Nucleotide positions are numbered relative to the major transcription start site $(+1)$ defined in the published DNA sequence. (b) Quantitative ChIP analysis of the occupancy of AcH3K9 after stimulation with $10 \mathrm{mM}$ butyrate acid in different regions of the MFG-E8 promoter. The results are expressed as the percentage of immunoprecipitate over total input DNA relative to glyceraldehyde-3-phosphate dehydrogenase (GAPDH). Values are shown as the mean and standard deviation of results from more than three independent experiments. ${ }^{*} P<0.05,{ }^{* *} P<0.01 \mathrm{vs}$ control (non-stimulated cells). (c) Time-dependent mRNA expression of MFG-E8 in NCI-H716 cells after stimulation with $10 \mu \mathrm{M}$ trichostatin $\mathrm{A}$, which also functions as a histone deacetylases (HDACs) inhibitor. All signals were standardized to the intensity of GAPDH. ${ }^{* *} P<0.01$ vs control (non-stimulated cells).

in the concentrations of stool organic acids were evaluated (Figure 1d, lower panel). The concentration of butyric acid showed a significant decrease with DSS treatment, such as that observed in WT mice, whereas that of the other organic acids did not change with or without DSS treatment.

\section{Butyric Acid Enema During Acute Phase of Experimental Colitis Attenuates Inflammation}

WT and MFG-E8KO mice were administered saline or $100 \mathrm{mM}$ butyric acid enemas for 10 days, ${ }^{10}$ during which they also received 2.5\% DSS in drinking water on the first 7 days (Figure 4a). As shown in Figure 4b, in WT mice that received DSS and saline enemas, body weight loss of $\sim 12 \%$ commenced on day 6 and continued to day 10, while those administered butyric acid enemas showed significant attenuation of body weight loss from day 6 , which reached only $5 \%$ on day 10 . Furthermore, MFG-E8KO mice that received saline enemas showed body weight loss of $\sim 16 \%$ that commenced on day 6 and continued to day 10, similar to WT mice with saline enemas. MFG-E8KO mice treated with butyric acid enemas were also prone to attenuation of body weight loss as seen with the control until day 7 , although the difference did not reach statistical significance on day 10. Colon samples dissected from WT mice with butyric acid enemas showed increased length as compared with those from saline-treated WT mice (Figure 4c).
On the other hand, representative dissected colon specimens from MFG-E8KO butyric acid-treated mice were similar in length as those from MFG-E8KO saline-treated DSS mice. Histological examinations also showed that inflammation, extent of injury, and crypt damage were significantly decreased in WT mice treated with butyric acid enemas (Figure 4d). Total histological scores for the dissected colon samples from WT mice treated with butyric acid enemas were also markedly lower than those from the other groups. Next, to investigate the effects of butyric acid enemas on MPO activity and proinflammatory cytokine production, proteins were extracted from colonic tissues, and MPO, TNF $\alpha$, IL-1 $\beta$, IL-6, and Mip-2 contents were measured (Figure $4 \mathrm{e}$ and Supplementary Figure 1). Notably, those were decreased in the butyric acidtreated mice, but not in WT mice in the DSS groups treated with saline. As for MFG-E8KO mice, those treated with butyric acid enemas also tended to show lower cytokine levels than untreated mice, although the differences were not significant. Finally, we measured MFG-E8 contents in colonic tissues (Figure 4f). As expected, DSS-treated WT mice given butyric acid enemas showed significantly upregulated MFG-E8 in the tissue contents as compared with those treated with saline enemas. Taken together, these results indicate that butyric acid has significant anti-inflammatory effects on DSS-induced experimental colitis via, at least in part, MFG-E8. 

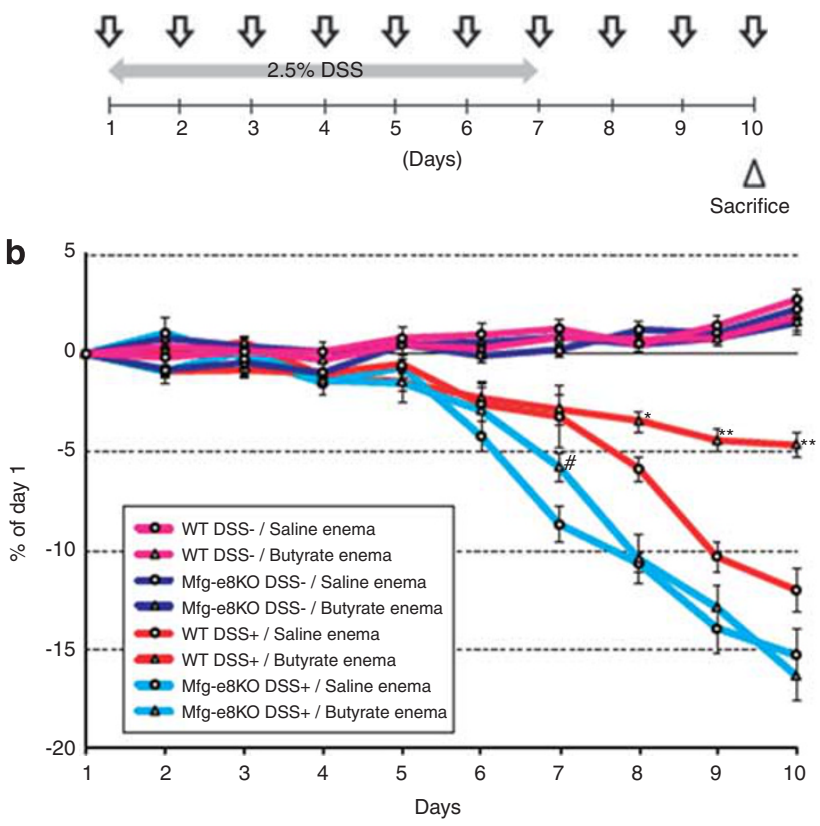

C

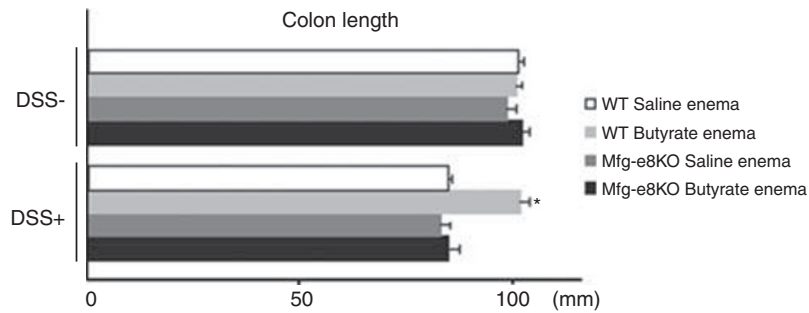

d
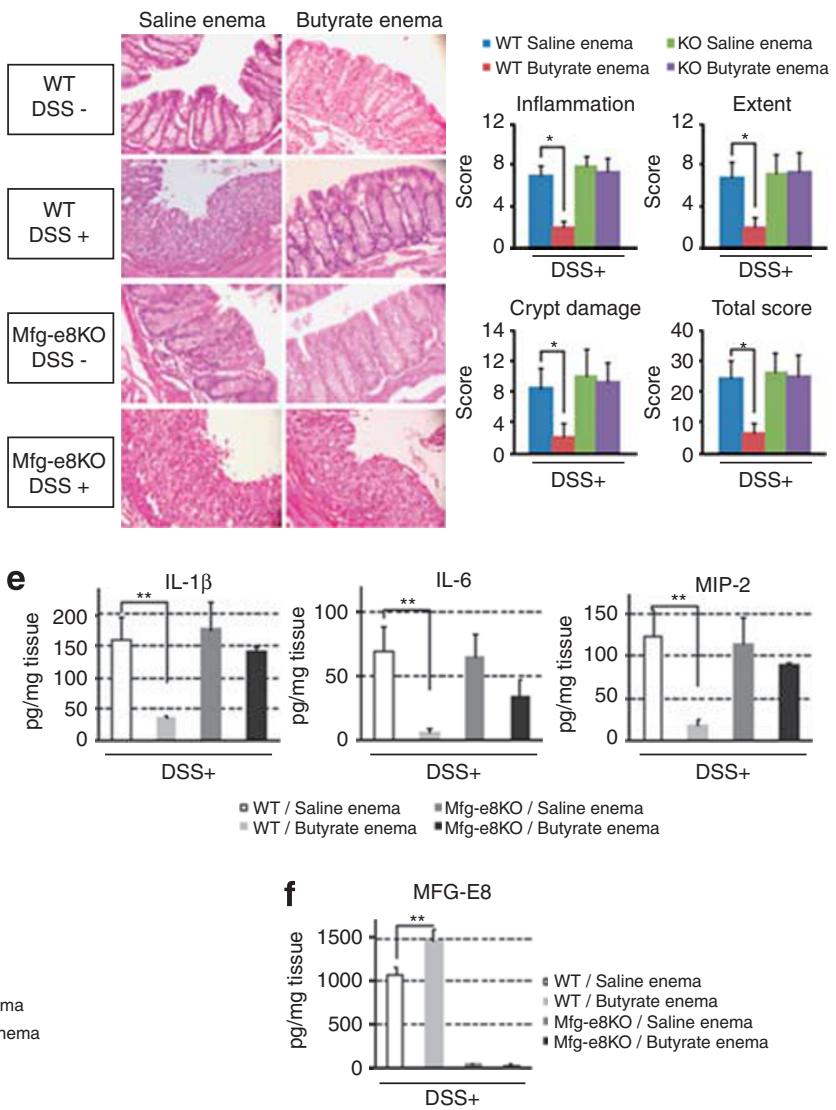

Figure 4 Intrarectal administration of butyrate acid protected mice from dextran sodium sulfate (DSS)-induced colitis via milk fat globule-epidermal growth factor 8 (MFG-E8). (a) Protocol for experimental colitis and treatment with $100 \mathrm{mM}$ butyrate. (b) Effects of butyrate on body weight changes in DSS-treated wild-type (WT) and MFG-E8 ${ }^{-1-}$ mice. Data are expressed as serial changes in the percentage of weight change during DSS administration. ${ }^{*} P<0.05,{ }^{*} P<0.01$ vs DSS-treated WT mice that received saline enemas, ${ }^{\#} P<0.05$ vs DSS-treated MFG-E8 ${ }^{-1-}$ mice that received saline enemas. Error bars indicate SEM values ( $n=5$ mice per group). (c) Effects of butyric acid enemas on colon length in mice with or without DSS treatment. Error bars indicate the standard error of mean values. ${ }^{*} P<0.05$ vs DSS-treated WT mice that received saline enemas. (d) Representative histological images and histological scores of colonic samples dissected from experimental mice. ${ }^{*} P<0.05$ vs DSS-treated WT mice that received saline enemas. Error bars indicate standard error of mean (s.e.m.) values ( $n=5$ mice per group). (e) Proinflammatory cytokines in colonic tissue contents of DSS-treated mice with or without intrarectal administration of butyrate acid. Cytokine contents were evaluated using enzyme-linked immunosorbent assay (ELISA). Error bars indicate the standard error of mean values independently obtained from five mice. ${ }^{* *} P<0.01$ vs DSS-treated WT mice that received saline enemas. (f) Effects of butyrate acid on MFG-E8 in DSS-treated mice. MFG-E8 contents in colonic tissues were evaluated using ELISA. Error bars indicate the standard error of mean values independently obtained from five mice. ${ }^{*} P<0.01$ vs DSS-treated WT mice with saline enema. IL, interleukin; $\mathrm{KO}$, knockout; MIP-2, macrophage inflammatory protein 2.

\section{DISCUSSION}

MFG-E8 was originally described as a factor in efficient phagocytosis of apoptotic cells for maintaining immune homeostasis. ${ }^{13,27}$ Apart from this conventional role, diverse functions have also been reported in several cellular and immune-mediated disorders. ${ }^{14,15}$ We previously found that the anti-inflammatory role of recombinant MFG-E8 protein in DSS-induced colitis was exerted by modulating LPSinduced innate-immune responses in macrophages. ${ }^{28}$ Also, Chogle et al. ${ }^{29}$ demonstrated that MFG-E8KO mice develop more severe crypt-epithelial injury during the onset of DSSinduced acute colitis, ${ }^{29}$ whereas a recent study revealed the anti-inflammatory effects of recombinant MFG-E8 protein in a mouse model of ischemia and reperfusion-induced intestinal injuries. ${ }^{16}$ Thus, a beneficial effect of MFG-E8 on downregulation of inflammation has been shown. In this study, we demonstrated its anti-inflammatory function via modulation of butyric acid during acute colitis.

Butyric acid is a molecule with a variety of effects, particularly towards colonic epithelial physiology and disease. ${ }^{6}$ Alone or in combination with other SCFAs, it has been used as therapy for a number of diseases characterized by colonic mucosal inflammation including UC. The protective effects of butyric acid in patients affected by these diseases have been 
proposed to result predominantly from influencing the energy availability in colonocytes. However, the cellular signaling events that mediate the protective effects of butyric acid in inflammatory bowel disease have not been fully elucidated.

The ability to produce butyrate acid is widely distributed among Gram-positive anaerobic bacteria that inhabit the human colon. ${ }^{30}$ Information regarding the phylogenetic diversity of butyric acid producers has been obtained primarily from cultural studies that used DNA sequence analysis and sequence-based detection methods. Two of the most important groups appear to be the clostridial clusters IV and XIVa, ${ }^{31,32}$ also known as the Clostridium leptum and coccoides groups, respectively. Frank et al. ${ }^{33}$ investigated mucosal biopsy specimens obtained from Crohn's disease (CD) and UC patients, and found decreases in the populations of clostridial clusters IV and XIVa. ${ }^{33}$ Using mice with DSS-mediated colitis, a model of colitis resembling human IBD, we also confirmed that DSS treatment in both WT and MFG-E8KO strains led to relative decreases in the Ruminococcaceae and Lachnospiraceae populations, which belong to the clostridial IV and XIVa groups, respectively. In another study, colonization of gnotobiotic mice by a defined mix of Clostridium strains provided an environment rich in transforming growth factor- $\beta$, and also affected Foxp $3^{+}$Treg number and function in the colon, ${ }^{34}$ whereas colitis symptoms and histological disease characteristics were significantly suppressed in Clostridium-abundant mice subjected to DSS treatment as compared with the control mice. The present $16 \mathrm{~S}$ rRNA analysis showed that the bacterial composition was not significantly different between WT and MFG-E8KO mice without DSS treatment. On the other hand, MFG-E8KO mice with DSS treatment tended to show decreases in the clostridial cluster IV and XIVa groups as compared with the control mice. These findings may explain, at least in part, why DSS-induced colonic inflammation in MFG-E8KO mice was more severe than that in WT mice.

In this study, we intrarectally injected butyric acid into mice that received DSS treatment, and found that the robust induction of MPO activity and proinflammatory cytokines during the acute phase of DSS-induced colitis was significantly downregulated by those injections. On the other hand, a less protective effect was seen in MFG-E8KO mice, with no significant differences found regarding bacterial composition and concentration changes of butyrate acid between WT and MFG-E8KO mice with DSS treatment. Therefore, these results support the concept of an anti-inflammatory effect of butyric acid via modulation of MFG-E8 and indicate a therapeutic benefit from intrarectal administration for intestinal inflammation.

Most human interventional studies with butyrate acid administration presented thus far have focused on overall clinical outcome parameters in patients with diversion colitis and active UC. ${ }^{9-11}$ UC is a chronic inflammatory disorder of the colon that is characterized by alternating periods of flareups and quiescent disease. ${ }^{35,36}$ It is generally considered that the disorder is the result of an exaggerated intestinal host response against luminal bacteria or their components, particularly in genetically susceptible individuals. In active UC patients, rectal enemas containing butyrate have been shown to be effective in reducing clinical symptoms and overall inflammatory scores, although not all studies

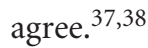

DNA methylation is one of the most commonly occurring epigenetic events in the mammalian genome and an important regulator of gene transcription. ${ }^{39,40}$ Increased DNA methylation in the promoter region of a gene leads to reduced expression, while the $\mathrm{CpG}$ island susceptible to methylation exists at around $-1000 \mathrm{bp}$ upstream of the promoter region of MFG-E8. Zhao et al. ${ }^{17}$ recently reported that MFG-E8 mRNA and protein expressions in colonic mucosal biopsy specimens from UC patients were lower than in those from healthy controls, and that MFG-E8 expression was inversely correlated with mucosal inflammatory and clinical disease activities in those patients. We speculate that hypermethylation of these promoter regions causes inactivation of MFG-E8 gene expression. Although butyric acid enema treatments generally have positive effects in UC patients, individual responses vary, as noted above. Therefore, the anti-inflammatory effect of MFG-E8 induced by butyric acid may not occur in some patients with potential inactivation of MFG-E8. As a result, it is necessary to evaluate the relationships of methylation state of the MFG-E8 promoter region with clinical symptoms related to inflammatory bowel disease.

In conclusion, the present findings showed a beneficial anti-inflammatory role of intrarectal butyrate-mediated MFG-E8 in a murine model of colitis. We also recently reported the anti-inflammatory effects of recombinant MFG-E8 protein for experimental colitis in mice. ${ }^{20} \mathrm{How}$ ever, several issues require further clarification before clinical use. As butyric acid significantly upregulates MFG-E8 expression, its effects with regard to physiological, immunological, and clinical aspects must also be evaluated in future studies.

Supplementary Information accompanies the paper on the Laboratory Investigation website (http://www.laboratoryinvestigation.org)

\section{ACKNOWLEDGEMENTS}

We thank Dr Shigekazu Nagata (Kyoto University, Japan) for kindly gifting the MFG-E8 ${ }^{-1}$ - mice, Ms Eri Sutoh, Ms Rika Tohma, and Ms Keiko Masuzaki (Shimane University, Japan) for their technical assistance, and the members of our laboratory for the helpful discussions. This study was supported in part by a grant (No. 23791033) from the Ministry of Education, Science, Sport, and Culture of Japan.

\section{DISCLOSURE/CONFLICT OF INTEREST}

The authors declare no conflict of interest. 
1. Garrett WS, Gordon Jl, Glimcher LH. Homeostasis and inflammation in the intestine. Cell 2010;140:859-870.

2. Kau AL, Ahern PP, Griffin NW, et al. Human nutrition, the gut microbiome and the immune system. Nature 2011;474:327-336.

3. Neish AS. Microbes in gastrointestinal health and disease. Gastroenterology 2009;136:65-80.

4. Round JL, Mazmanian SK. The gut microbiota shapes intestinal immune responses during health and disease. Nat Rev Immunol 2009;9:313-323.

5. Cook Sl, Sellin JH. Review article: short chain fatty acids in health and disease. Aliment Pharmacol Ther 1998;12:499-507.

6. Hamer HM, Jonkers $D$, Venema $K$, et al. Review article: the role of butyrate on colonic function. Aliment Pharmacol Ther 2008;27:104-119.

7. Butzner JD, Parmar R, Bell CJ, et al. Butyrate enema therapy stimulates mucosal repair in experimental colitis in the rat. Gut 1996;38:568-573.

8. Andoh A, Bamba T, Sasaki M. Physiological and anti-inflammatory roles of dietary fiber and butyrate in intestinal functions. J Parenter Enteral Nutr 1999:23:S70-S73.

9. Harig JM, Soergel $\mathrm{KH}$, Komorowski RA, et al. Treatment of diversion colitis with short-chain-fatty acid irrigation. N Engl J Med 1989;320:23-28.

10. Scheppach W, Sommer H, Kirchner T, et al. Effect of butyrate enemas on the colonic mucosa in distal ulcerative colitis. Gastroenterology 1992;103:51-56.

11. Vernia P, Annese V, Bresci G, et al. Topical butyrate improves efficacy of 5-ASA in refractory distal ulcerative colitis: results of a multicentre trial. Eur J Clin Invest 2003;33:244-248.

12. Stubbs JD, Lekutis $C$, Singer $\mathrm{KL}$, et al. $\mathrm{CDNA}$ cloning of a mouse mammary epithelial cell surface protein reveals the existence of epidermal growth factor-like domains linked to factor VIII-like sequences. Proc Natl Acad Sci USA 1990;87:8417-8421.

13. Hanayama R, Tanaka M, Miwa K, et al. Identification of a factor that links apoptotic cells to phagocytes. Nature 2002;417:182-187.

14. Komura $\mathrm{H}$, Miksa $M$, Wu $R$, et al. Milk fat globule epidermal growth factor-factor VIII is down-regulated in sepsis via the lipopolysaccharide-CD14 pathway. J Immunol 2009;182:581-587.

15. Kranich J, Krautler NJ, Falsig J, et al. Engulfment of cerebral apoptotic bodies controls the course of prion disease in a mouse straindependent manner. J Exp Med 2010;207:2271-2281.

16. Cui T, Miksa M, Wu R, et al. Milk fat globule epidermal growth factor 8 attenuates acute lung injury in mice after intestinal ischemia and reperfusion. Am J Respir Crit Care Med 2010;181:238-246.

17. Zhao QJ, Yu YB, Zuo XL, et al. Milk fat globule-epidermal growth factor 8 is decreased in intestinal epithelium of ulcerative colitis patients and thereby causes increased apoptosis and impaired wound healing. Mo Med 2012;18:497-506.

18. Aziz M, Jacob A, Matsuda A, et al. Review: milk fat globule-EGF factor 8 expression, function and plausible signal transduction in resolving inflammation. Apoptosis 2011;16:1077-1086.

19. Kusunoki R, Ishihara S, Aziz M, et al. Roles of milk fat globule-epidermal growth factor 8 in intestinal inflammation. Digestion 2012;85:103-107.

20. Otani A, Ishihara S, Aziz MM, et al. Intrarectal administration of milk fat globule epidermal growth factor-8 protein ameliorates murine experimental colitis. Int J Mol Med 2012;29:349-356.
21. Bibiloni $\mathrm{R}$, Simon MA, Albright $C_{\text {, }}$ et al. Analysis of the large bowe microbiota of colitic mice using PCR/DGGE. Lett Appl Microbiol 2005:41:45-51.

22. Mishiro $\mathrm{T}$, Ishihara $\mathrm{K}$, Hino $\mathrm{S}$, et al. Architectural roles of multiple chromatin insulators at the human apolipoprotein gene cluster. EMBO J 2009;28:1234-1245.

23. Dzierzewicz Z, Orchel A, Weglarz L, et al. Changes in the cellular behaviour of human colonic cell line Caco-2 in response to butyrate treatment. Acta Biochim Pol 2002;49:211-220.

24. Portela A, Esteller M. Epigenetic modifications and human disease. Nat Biotechnol 2010;28:1057-1068.

25. Marks PA, Richon VM, Miller T, et al. Histone deacetylase inhibitors. Adv Cancer Res 2004;91:137-168.

26. Mariadason JM. HDACs and HDAC inhibitors in colon cancer Epigenetics 2008;3:28-37.

27. Hanayama $\mathrm{R}$, Tanaka M, Miyasaka $\mathrm{K}$, et al. Autoimmune disease and impaired uptake of apoptotic cells in MFG-E8-deficient mice. Science 2004:304:1147-1150.

28. Aziz MM, Ishihara $S$, Mishima $Y$, et al. MFG-E8 attenuates intestinal inflammation in murine experimental colitis by modulating osteopontin-dependent alphavbeta3 integrin signaling. J Immunol 2009;182:7222-7232.

29. Chogle A, Bu HF, Wang X, et al. Milk fat globule-EGF factor 8 is a critical protein for healing of dextran sodium sulfate-induced acute colitis in mice. Mol Med 2011:17:502-507.

30. Barcenilla A, Pryde SE, Martin JC, et al. Phylogenetic relationships of butyrate-producing bacteria from the human gut. Appl Environ Microbiol 2000;66:1654-1661.

31. Hold GL, Pryde SE, Russell VJ, et al. Assessment of microbial diversity in human colonic samples by $16 \mathrm{~S}$ rDNA sequence analysis. FEMS Microbiol Ecol 2002;39:33-39.

32. Fava F, Danese S. Intestinal microbiota in inflammatory bowel disease: friend of foe? World J Gastroenterol 2011;17:557-566.

33. Frank St DN, Amand AL, Feldman RA, et al. Molecular-phylogenetic characterization of microbial community imbalances in human inflammatory bowel diseases. Proc Natl Acad Sci USA 2007;104: 13780-13785.

34. Atarashi K, Tanoue $\mathrm{T}$, Shima $\mathrm{T}$, et al. Induction of colonic regulatory T cells by indigenous Clostridium species. Science 2011;331:337-341.

35. Podolsky DK. Inflammatory bowel disease. N Engl J Med 2002;347:417-429.

36. Xavier RJ, Podolsky DK. Unravelling the pathogenesis of inflammatory bowel disease. Nature 2007;448:427-434.

37. Scheppach W. Treatment of distal ulcerative colitis with short-chain fatty acid enemas. A placebo-controlled trial. German-Austrian SCFA Study Group. Dig Dis Sci 1996:41:2254-2259.

38. Steinhart AH, Hiruki T, Brzezinski $A$, et al. Treatment of left-sided ulcerative colitis with butyrate enemas: a controlled trial. Aliment Pharmacol Ther 1996;10:729-736.

39. Black JC, Whetstine JR. Chromatin landscape: methylation beyond transcription. Epigenetics 2011;6:9-15.

40. Deaton AM, Bird A. CpG islands and the regulation of transcription Genes Dev 2011:25:1010-1022. 